Goldschmidt 2021 Abstract

https://doi.org/10.7185/gold2021.6892

\section{Controlling Crystallization by Surface Topography}

THOMAS HENRY DUNN, PHILLIP ALAN LEE, FIONA MELDRUM AND ALEXANDER KULAK

University of Leeds

Presenting Author: cm15td@leeds.ac.uk

The addition of nucleants to a crystallisation reaction provides a means of controlling the size and structure of the crystals produced, where they form, and the induction time. However, with the exception of epitaxial matching, there is little rationale for the identification of effective nucleants for many systems. In this work, we explore the potential of using surface topography to direct nucleation, where evidence is scattered across the literature that nucleation is promoted in features such as pits and scratches. Substrates patterned with a network of natural surface cracks are created in this study, where these spontaneously form in materials whose surfaces are under strain. These cracked substrates are then coated with a thin film of a noble metal and chemically functionalised to enable control over the surface chemistry. Controlling both the topography and surface chemistry of these substrates is shown to be highly effective in directing the nucleation of a range of substances including various metal carbonates (Figures $1 \& 2$ ). A high density of crystals preferentially forms at the cracks, and control over calcium carbonate polymorph can be achieved according to the selection of the surface chemistry. The origin and generality of this phenomenon is explored to determine how the topography and surface chemistry can be altered to promote the nucleation of a specific crystal. Controlling surface topography is therefore a valuable and frequently overlooked tool in controlling nucleation and can potentially be used in the design of effective nucleants.

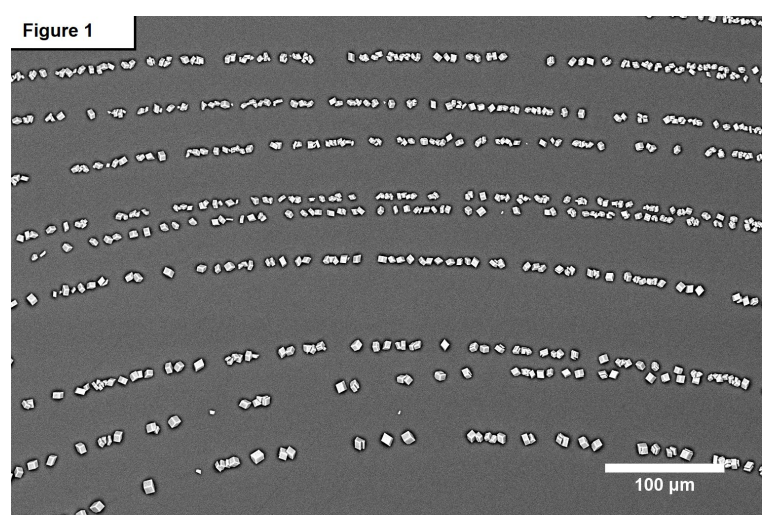

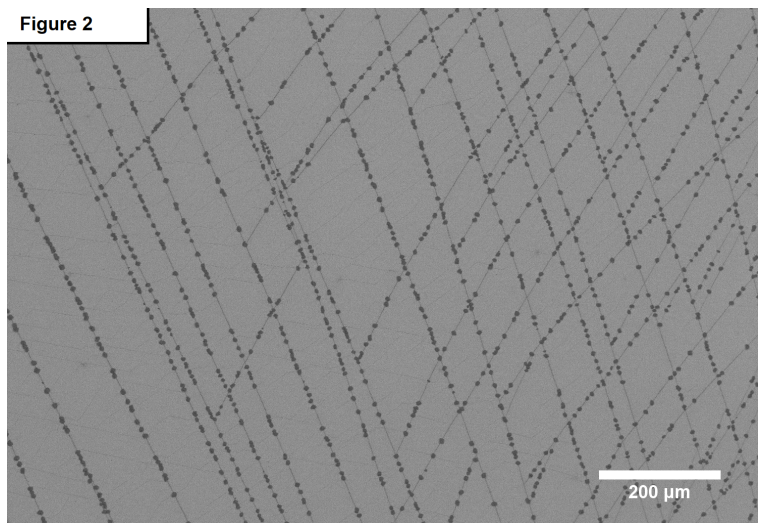

